\title{
A Compreensão da Gratidão e Teoria da Mente em Crianças de 5 Anos
}

\author{
The Understanding of Gratitude and Theory of Mind in 5-Year-Olds
}

\author{
Lia Beatriz de Lucca Freitas ${ }^{*}, a$, Marion O’Brien ${ }^{b}$, Jackie A. Nelson ${ }^{b} \&$ Stuart Marcovitch $^{b}$ \\ ${ }^{a}$ Universidade Federal do Rio Grande do Sul, Porto Alegre, Brasil \\ $\&{ }^{b}$ University of North Carolina, Greensboro, USA
}

\begin{abstract}
Resumo
Examinaram-se relações entre compreensão da gratidão (GRA) e desenvolvimento de uma teoria da mente (TOM) com 228 crianças norteamericanas (53\% meninas) de 5 anos. Testaram-se as seguintes hipóteses: (a) não há diferença de sexo em GRA ou TOM, (b) as crianças com melhor desempenho nas tarefas de TOM têm melhor GRA, (c) TOM é condição necessária para GRA. Utilizaram-se três tarefas para avaliar TOM: consideração da perspectiva visual, crença falsa de primeira e de segunda ordem. Contaram-se às crianças duas histórias sobre gratidão. Avaliou-se GRA a partir de suas respostas a perguntas feitas após a leitura de cada história. As duas primeiras hipóteses foram confirmadas. A hipótese de que TOM seria condição necessária para GRA não encontrou suficiente suporte empírico.
\end{abstract}

Palavras-chave: Gratidão, teoria da mente, desenvolvimento, crianças.

\begin{abstract}
We examined relations between the understanding of gratitude (GRA) and the development of a theory of mind (TOM). The study was done with 2285 -year-old North American children ( $53 \%$ female). We tested the following hypotheses: (a) there are no sex differences in GRA or TOM, (b) children who perform better on TOM tasks have better GRA, (c) TOM is a necessary condition for GRA. We used three tasks to evaluate TOM: visual perspective taking, first-order false belief, and second-order false belief. The children were read two vignettes about gratitude. GRA was evaluated based on children's responses to questions asked after each vignette was read. The first two hypotheses were supported. The hypothesis that TOM would be a necessary condition for GRA did not receive sufficient empirical support.

Keywords: Gratitude, theory of mind, development, children.
\end{abstract}

A gratidão surge, em geral, quando uma pessoa - o(a) beneficiário(a) - recebe um benefício (presente, favor ou ajuda) de uma outra - o(a) benfeitor(a). Na literatura, a capacidade de experienciar um sentimento positivo, quando se recebe um benefício, tem sido considerada condição necessária à gratidão (Bonnie \& de Waal, 2004; Komter, 2004; Piaget, 1954). No entanto, a gratidão é um sentimento reativo tanto ao benefício recebido quanto ao benfeitor (Baumgarten-Tramer, 1938; McConnell, 1993). Assim, como afirmaram Bonnie e de Waal (2004), “em-

* Esta pesquisa recebeu apoio financeiro da Eunice Kennedy Shriver National Institute of Child Health and Human Development, processo 5R01HD050806, e da Coordenação de Aperfeiçoamento de Pessoal de Nível Superior (CAPES), através de bolsa de estudos à primeira autora para realização de Estágio Sênior no Exterior, processo BEX 2038/09-9. Os autores gostariam também de agradecer a Parita Vithlani pela transcrição das entrevistas e a Kaley Stone por seu auxílio na codificação das entrevistas sobre gratidão.

Address: Laboratório de Psicologia e Epistemologia Genética, Universidade Federal do Rio Grande do Sul, Rua Ramiro Barcelos, 2600, sala 118, Porto Alegre, RS, Brasil 90035-003. E-mail: lapege@lapege.com.br bora um apreço aos favores recebidos seja um componente necessário da gratidão, a resposta emocional de sentir-se bem por si só não é suficiente" (pp. 221-222).

A avaliação das intenções do benfeitor é também um elemento essencial da gratidão. De acordo com McConnell (1993), é a natureza gratuita da ação generosa do benfeitor que a torna digna de gratidão. Isto significa que o benefício foi dado livre (sem o intuito de colocar o beneficiário em dívida) e gratuitamente (sem retribuição requerida).

Ao atribuir boas intenções ao benfeitor, o beneficiário passa a valorizar não apenas o benefício, mas também o próprio benfeitor. Como resultado, o beneficiário sente uma dívida em relação ao benfeitor (uma "dívida de gratidão") e uma necessidade de retribuir o benefício (Bonnie \& de Waal, 2004; Piaget, 1965/1977). Este sentimento de obrigatoriedade de retribuir o favor ao benfeitor prévio é, portanto, também um componente-chave da gratidão (McConnell, 1993). Este sentimento gera uma espiral ascendente de comportamentos pró-sociais de ajuda e apoio mútuos (McCullough, Kilpatrick, Emmons, \& Larson, 2001).

Receber presentes, favores e ajuda faz parte da vida das crianças. Embora relativamente poucos estudos fo- 
ram realizados com crianças (Froh, 2010; McCullough et al., 2001; Pieta \& Freitas, 2009), existem algumas evidências de que as crianças são capazes de vivenciar, expressar e compreender a gratidão.

$O$ desenvolvimento da gratidão na infância. A literatura disponível sobre o assunto não é extensa nem homogênea, visto que diversos aspectos do desenvolvimento da gratidão foram examinados. O estudo pioneiro de Baumgarten-Tramer (1938) com crianças e adolescentes de 7 a 15 anos indicou uma clara evolução da gratidão concreta à conectiva. A gratidão concreta caracteriza-se pelo fato de que o beneficiário retribui com algo que tem valor para ele e não necessariamente para o benfeitor. $\mathrm{Na}$ gratidão conectiva, pelo contrário, o beneficiário busca retribuir o favor com algo que agrade ou vá ao encontro das necessidades do benfeitor. Segundo BaumgartenTramer, esta evolução deve-se ao fato de que as crianças mais velhas e os adolescentes, sendo menos egocêntricos, são mais capazes de considerar o ponto de vista dos outros. A gratidão "verbal" (por exemplo, dizer "obrigado") foi expressa em todas as idades. Da mesma forma, Freitas, Pieta e Tudge (2011) verificaram um decréscimo na frequência de gratidão concreta e um aumento do tipo conectiva com o avanço da idade. Não foram encontradas diferenças significativas na forma como meninos e meninas expressaram gratidão. Estes estudos indicam que crianças e adolescentes utilizam outras formas para expressar gratidão, além das formas de polidez ensinadas pelos adultos. Os resultados encontrados são importantes, uma vez que um dos maiores desafios enfrentados em pesquisas com crianças é a distinção entre um genuíno sentimento de gratidão e a polidez (Bono \& Froh, 2009; Gordon, Musher-Eizenman, Holub, \& Dalrymple, 2004).

Alguns pesquisadores detiveram-se exatamente nessas formas de polidez, examinando a tendência das crianças a agradecer, quando recebem um presente. Gleason e Weintraub (1976) observaram que apenas $21 \%$ das crianças com menos de 6 anos agradeceram a um adulto que lhes deu balas no dia de Halloween, enquanto mais de $80 \%$ das crianças de 10 anos ou mais agradeceram nessa mesma situação. Este dado chamou nossa atenção, visto que, desde cedo, pais e professores ensinam às crianças a agradecer, quando recebem um presente ou ajuda. Becker e Smenner (1986) estudaram o agradecimento verbal espontâneo de crianças de 3 e 4 anos frente a uma recompensa. Eles constataram que as crianças agradeceram mais a um adulto que a uma outra criança. Este resultado sugere que existe uma atividade interpretativa da criança daquilo que lhe é ensinado, visto que pais e professores não fazem essa distinção entre adultos e crianças. Talvez, as crianças agradeçam mais aos adultos, porque são eles que prescrevem essa norma (Piaget, 1932/1992).

Gordon et al. (2004) investigaram a que as crianças são gratas, comparando relatos de crianças de 4-8 anos e 912 anos, antes e depois do atentado de 11 de setembro. Os resultados sugeriram que as crianças de 9-12 anos têm mais gratidão às pessoas (pais, amigos, professores, etc.) do que as de 4-8 anos, as quais se referiram com maior frequência a objetos materiais, o que indica, segundo esses autores, uma diminuição do egocentrismo e um aumento de interesse pelas relações interpessoais com a idade. Isto pode explicar, em parte, por que as crianças pequenas agradecem às pessoas menos que as crianças mais velhas, quando recebem um presente. Os resultados também indicaram uma diferença de gênero: (a) os relatos das meninas apresentaram mais temas do que os dos meninos e (b) as meninas mostraram-se mais gratas às pessoas, enquanto os meninos, a objetos materiais. Segundo Gordon et al. (2004) esses dados sugerem que as diferenças entre meninos e meninas na forma e importância dada às relações interpessoais influenciam a gratidão. As meninas passam mais tempo com as pessoas que os meninos. Além disto, há mais intimidade nas relações de amizade entre meninas, enquanto os grupos sociais dos meninos caracterizam-se mais por relações hierárquicas e competitivas.

Outros pesquisadores não estudaram diretamente a gratidão, mas investigaram a compreensão das crianças sobre diversos sentimentos complexos, dentre os quais a gratidão (Harris, Olthof, Meerum Terwogt, \& Hardman, 1987; Russell \& Paris, 1994). No entanto, os resultados não foram conclusivos quanto ao processo de aquisição desses conceitos. Harris et al. (1987) sugeriram que a compreensão desses sentimentos ocorreria de forma abrupta, após os 7 anos de idade. Pelo contrário, para Russell e Paris, essa aquisição ocorreria de forma gradual: (a) conhecimento da valência (positiva ou negativa) do sentimento e (b) conhecimento de situações específicas que o eliciam. Mesmo crianças de 4 anos foram capazes de relacionar sentimentos positivos a situações que desencadeiam gratidão. Neste mesmo sentido, Graham e Barker (1990) constataram que crianças de 4 a 12 anos, independentemente de sua idade, acreditavam que um estudante que havia recebido ajuda de sua professora para resolver um problema sentiria mais gratidão que aquele que não havia recebido ajuda nessa mesma situação. Todavia, tanto Harris et al. (1987) quanto Russell e Paris (1994) enfatizaram a importância de se incluir sentimentos complexos em estudos com crianças pré-escolares e não apenas emoções básicas (alegria, tristeza, medo e raiva).

Outro importante aspecto investigado foi a relação entre gratidão $\mathrm{e}$ intencionalidade. $\mathrm{O}$ beneficiário sente gratidão quando o benefício foi dado intencionalmente (McConnell, 1993). A avaliação da intenção do benfeitor parece desenvolver-se ao longo da infância. No estudo de Graham (1988), as crianças menores (5-6 e 7-8 anos), avaliaram que o beneficiário retribuiria o favor, independentemente da intenção do benfeitor; as crianças de 10 anos, pelo contrário, julgaram a retribuição apropriada apenas quando o benefício havia sido dado intencionalmente. Os resultados do estudo de Freitas, Silveira e Pieta (2009a) com crianças de 5 a 12 anos também sugeriram que a avaliação das intenções do benfeitor é um compo- 
nente essencial da gratidão: apenas a ação generosa desencadeia sentimentos positivos no beneficiário.

Castro, Rava, Hoefelmann, Pieta e Freitas (2011) investigaram se as crianças sentem obrigação de retribuir um favor, enfocando especialmente as suas justificativas. Os resultados indicaram uma evolução na forma como as crianças concebem a obrigação de retribuir: embora nenhuma criança de 5-6 anos tenha mencionado a retribuição do favor, as crianças maiores de 7 anos o fizeram. Para a maioria das crianças mais velhas, a razão para retribuir o favor era evitar o juízo alheio negativo. No entanto, para algumas (e somente crianças de 11 ou 12 anos) a retribuição de um favor a um benfeitor prévio é um valor moral.

Em síntese, os dados disponíveis indicam que existe um desenvolvimento da gratidão na infância desde os anos pré-escolares, embora a trajetória desse desenvolvimento seja ainda uma questão a ser investigada (Froh \& Bono, 2008). Além disso, alguns resultados de pesquisa sugerem que existe uma relação entre o desenvolvimento da gratidão e o desenvolvimento de habilidades sociocognitivas (Baumgarten-Tramer, 1938; Gordon et al., 2004; Graham, 1988).

Gratidão e teoria da mente. McAdams e Bauer (2004) levantaram a hipótese de que sentir gratidão requer o desenvolvimento de uma teoria da mente. Teoria da mente é a forma como os pesquisadores têm-se referido à capacidade adquirida por crianças pré-escolares de entender estados mentais (desejos, sentimentos, crenças e intenções) de outras pessoas, assim como delas mesmas (Flavell, Miller, \& Miller, 2002), e a consciência de que as percepções nem sempre refletem a realidade (para mais detalhes, ver Jou \& Sperb, 1999; Sperb \& Maluf, 2009). Esta capacidade é muito importante para a compreensão dos motivos e intenções de outras pessoas, facilitando o estabelecimento e a manutenção de relações interpessoais. Como afirmaram Roazzi e Santana (2008), esta é uma habilidade necessária "para qualquer compreensão sofisticada das peculiaridades que envolvem as relações sociais" (p. 444).

Investiga-se a aquisição dessas habilidades através de tarefas de crença falsa, as quais consistem, geralmente, em contar uma história à criança, na qual existem dados que permitem inferir que o protagonista tem uma crença diferente da realidade. Nas tarefas de crença falsa de primeira ordem, a criança deve entender que um personagem A tem uma crença falsa sobre a realidade. Para ser bem sucedida, a criança deve ser capaz de atribuir uma representação falsa da realidade a A. Nas tarefas de crença falsa de segunda ordem, a criança deve entender que um personagem A tem uma crença falsa sobre a crença de um personagem B. Em outras palavras, a criança precisa ser capaz de atribuir uma representação falsa a A sobre a representação de $\mathrm{B}$.

$\mathrm{Na}$ literatura, pode-se observar um crescente interesse em investigar as possíveis relações entre o desenvolvimento de uma teoria da mente e a compreensão de emo- ções e sentimentos. Por exemplo, Harwood e Farrar (2006) sugeriram que levar em conta a perspectiva afetiva de outros, particularmente a identificação de emoções que diferem daquelas da própria criança, depende de capacidades inerentes às tarefas que avaliam o desenvolvimento de uma teoria da mente. Neste mesmo sentido, de Rosnay, Pons, Harris e Morrell (2004) constataram que as crianças entendem crenças falsas antes de serem capazes de atribuir emoções com precisão. Da mesma forma, Wellman e Liu (2004) verificaram que uma compreensão inicial da mente (própria e dos outros) é necessária para a criança entender que um indivíduo pode sentir uma emoção, mas mostrar outra. Alguns estudos, pelo contrário, sugerem que a compreensão de emoções aparece antes e dá suporte ao desenvolvimento de uma teoria da mente (Bartsch \& Wellman, 1995; Blankson, O’Brien, Leerkes, Marcovitch, \& Calkins, in press). No Brasil, o estudo de Santana e Roazzi (2006) também sugere que a capacidade de predizer e justificar emoções básicas se desenvolve anteriormente a uma teoria da mente. No entanto, Cutting e Dunn (1999) haviam sugerido que a compreensão de emoções e uma teoria da mente se desenvolvem de forma paralela, mas posteriormente integram-se em um mesmo sistema sociocognitivo. Embora a maioria desses estudos tenha enfocado emoções básicas, alguns trataram de sentimentos complexos, tais como vergonha e constrangimento (e.g., Heerey, Keltner, \& Capps, 2003; Racine, Carpendale, \& Turnbull, 2007).

A hipótese de McAdams e Bauer (2004) parece plausível, uma vez que a avaliação da intenção do benfeitor é um elemento-chave da gratidão (Bonnie \& de Waal, 2004; Freitas et al., 2009a; Graham, 1988; McConnell, 1993). É também possível examinar esta hipótese, visto que alguns estudos abordaram a relação entre teoria da mente e sentimentos complexos. No entanto, até onde sabemos, esta hipótese ainda não tinha sido testada.

Neste estudo, buscou-se verificar empiricamente a hipótese de que a compreensão da gratidão em crianças pré-escolares está relacionada ao desenvolvimento de uma teoria da mente. Em primeiro lugar, previa-se que não haveria uma diferença entre meninos e meninas, tanto no que diz respeito à compreensão da gratidão quanto ao desenvolvimento de uma teoria da mente (neste artigo, denominada hipótese 1). Embora Gordon et al. (2004) tenham encontrado diferenças no que diz respeito a que meninos e meninas são gratos, não se encontrou ainda suficiente suporte empírico para a existência de diferenças entre os sexos em outros estudos (Freitas et al., 2011; Froh, Kashdan, Ozimkowski, \& Miller, 2009). Em segundo lugar, esperava-se que as crianças que tivessem um melhor desempenho em tarefas que avaliam o desenvolvimento de uma teoria da mente teriam uma melhor compreensão da gratidão (hipótese 2). Em terceiro lugar, se esta hipótese fosse confirmada, considerando-se a proposição de McAdams e Bauer (2004), examinar-se-ia também se uma teoria da mente é condição necessária para a compreensão da gratidão (hipótese 3 ). 


\section{Método}

\section{Participantes}

A amostra foi composta por 228 crianças americanas de 5 anos $(M=65,59$ meses; $D P=2,14)$, as quais participaram de um estudo mais amplo, cujo objetivo geral foi avaliar processos emocionais e cognitivos iniciais e identificar suas contribuições tanto para o funcionamento social quanto para o sucesso escolar de crianças préescolares. Aproximadamente $42 \%$ das crianças pertenciam a famílias de baixa-renda, $45 \%$ a famílias de renda média e $13 \%$ a famílias de renda alta. As mães de $57 \%$ das crianças tinham pelo menos quatro anos de educação de nível superior. Cinquenta e três por cento das crianças eram meninas; $60 \%$ eram euroamericanas, $33 \%$ afroamericanas e $7 \%$ de outras etnias.

\section{Procedimentos Gerais}

Recrutaram-se os participantes em pré-escolas de uma cidade com aproximadamente 230 mil habitantes, no sudeste dos Estados Unidos, através de cartas enviadas aos pais ou responsáveis das crianças. Os interessados em participar do estudo forneceram informações para contato e os pesquisadores, então, telefonaram para marcar uma visita ao laboratório. Durante a sessão no laboratório, as crianças foram filmadas, enquanto realizavam as tarefas. Sempre que as crianças solicitavam, as tarefas eram interrompidas. Durante a sessão, todas as crianças receberam um lanche e tiveram um espaço de tempo para brincar com suas mães ou seus pais. Os pais ou responsáveis dos participantes assinaram um termo de consentimento livre e esclarecido.

\section{Instrumentos}

Teoria da mente. Utilizaram-se três tarefas para avaliar o desenvolvimento de uma teoria da mente: (a) consideração da perspectiva visual, (b) crença falsa de primeira ordem e (c) crença falsa de segunda ordem.

Consideração da perspectiva visual. Esta tarefa avalia se a criança é capaz de levar em conta a perspectiva dos outros (Flavell, Everett, Croft, \& Flavell, 1981; Taylor, 1988), sendo organizada em dois níveis. No nível 1, a criança precisa apenas reconhecer que outra pessoa nem sempre vê o que ela mesma vê; no nível 2, ela deve diferenciar o seu próprio ponto de vista daquele de outra pessoa sobre um mesmo estímulo. Inicialmente, colocava-se na frente da criança horizontalmente sobre a mesa um cartão com o desenho de um coelho e alternava-se a sua posição, ora estando de cabeça para cima do lado da criança ora do lado do entrevistador (nível 2). A seguir, apresentava-se à criança um cartão que tinha o desenho de uma zebra, de um lado, e o desenho de um bode, do outro. O cartão era colocado sobre a mesa, verticalmente, entre a criança e o entrevistador. Perguntava-se à criança o que ela podia ver e qual animal o entrevistador estava vendo (nível 1). A criança recebeu 1 ponto para cada resposta correta sobre a perspectiva do entrevistador, e somaram-se as respostas corretas. O escore total da tarefa variou entre 0 e 3 .

Crença falsa de primeira ordem. Nesta tarefa, pede-se à criança para predizer o comportamento de uma boneca, quando esta boneca tem uma crença falsa sobre a localização de um objeto escondido. Uma primeira boneca coloca um objeto em uma caixa e sai da sala. Uma segunda boneca, então, retira o objeto dessa caixa e o coloca em uma outra caixa e também sai da sala. A primeira boneca retorna à sala e procura o objeto. O entrevistador faz várias perguntas à criança sobre o que a primeira boneca pensa e faz quando retorna à sala (Onde ela procurará o objeto? Onde ela pensa que está? Onde está realmente?). Esta prova foi apresentada duas vezes (com diferentes objetos). A criança recebeu 1 ponto para cada juízo correto sobre uma crença falsa. Cada vez que a prova foi apresentada, as perguntas "Onde a boneca procurará o objeto?" e "Onde a boneca pensa que está?" foram pontuadas. Assim, o escore total da tarefa variou entre 0 e 4.

Crença falsa de segunda ordem. Esta tarefa foi inicialmente desenvolvida por Perner e Wimmer (1985). Utilizou-se a versão proposta por Sullivan, Zaitchik e Tager-Flusberg (1994) por ser mais adequada à idade das crianças deste estudo. Contou-se uma história à criança com dois personagens: Patty e Sally. Sally prega uma peça em Patty escondendo o seu brinquedo no armário. Sally não sabe, porém, que Patty a viu esconder o brinquedo, olhando pela janela (versão masculina: Paul e Sam). Perguntou-se à criança uma série de questões sobre o conhecimento e as crenças dos dois personagens sobre o que aconteceu. Para responder corretamente, a criança deve levar em conta a ignorância de Sally sobre o conhecimento de Patty a respeito da localização real do objeto. Sete perguntas foram feitas à criança, sendo duas relativas a crenças falsas de segunda ordem. As crianças receberam 1 ponto para cada juízo correto, sendo que o escore total da tarefa variou entre 0 e 2 .

Gratidão. Para avaliar a compreensão da gratidão, contou-se às crianças duas pequenas histórias, traduzidas para o inglês, as quais haviam sido utilizadas em estudos com crianças brasileiras de 5 a 12 anos de idade (Freitas et al., 2009a, 2009b; Castro et al., 2011), tendo sido compreendidas mesmo pelas crianças menores.

História da gatinha. Contou-se à criança a seguinte história:

Nicky tinha uma gatinha. Um dia ela desapareceu. Tia Anne, que estava fazendo um bolo, disse: 'Não tem problema. Eu te ajudo a procurar tua gatinha.' Elas ficaram muito tempo procurando. Quando estava quase escuro, elas encontraram a gatinha. Tia Anne teve que jogar a massa do bolo fora e começar tudo de novo" (versão masculina: Nicky e Tia Anne).

Perguntaram-se à criança as seguintes questões: Como Nicky se sentiu? Por quê? Ela sentiu mais alguma coisa? 
Ela sentiu alguma coisa pela tia Anne? Por quê? Nicky deve ajudar tia Anne a fazer o bolo? Por quê?

História do sweater. Contou-se a seguinte história: “Em um dia de inverno, Danielle estava com frio. Jane, uma menina nova na escola, tinha um outro sweater em sua mochila. Então, ela emprestou esse sweater para Danielle" (versão masculina: David e John). Formularamse à criança às questões: Como Danielle se sentiu? Por quê? Ela sentiu mais alguma coisa? Ela sentiu alguma coisa pela Jane? Por quê? E Jane, como ela se sentiu? Por quê? Por que Jane emprestou o sweater? Após ter respondido às perguntas, foi dito à criança: "Esta história continua: Na semana seguinte, Jane esqueceu sua tesoura em casa. A professora tinha pedido que todos trouxessem tesoura, naquele dia. Danielle tem uma outra tesoura em sua mochila." Perguntou-se: Danielle deve emprestar a tesoura para Jane ou não? Por quê?

As histórias eram encenadas, sendo os personagens representados por bonecos (cerca de $5 \mathrm{~cm}$ ). As crianças foram filmadas, as entrevistas transcritas e, então, codificadas. Examinaram-se quatro componentes da gratidão: (a) sentimentos positivos, (b) relação com o benfeitor, (c) a obrigação (ou dever) de retribuir o favor, e (d) tipos de justificativa. A Tabela 1 apresenta os códigos e exemplos de respostas dos participantes (para mais detalhes, ver Freitas et al., 2009a, 2009b; Castro et al., 2011). O índice de concordância interjuízes, estimado pelo coeficiente Kappa, foi alto, tendo variado entre 0,82 e 1 na história da gatinha e entre 0,9 e 1 na história do sweater. A soma desses quatro componentes representa o escore total, o qual variou entre 0 e 6 .

Tabela 1

Códigos e Exemplos de Respostas dos Participantes nas Histórias de Gratidão

\begin{tabular}{|c|c|c|c|}
\hline Componentes & Descrição & Respostas (exemplos) & Escore \\
\hline \multicolumn{4}{|c|}{ História da Gatinha } \\
\hline Sentimentos positivos & $\begin{array}{l}\text { Não } \\
\text { Sim }\end{array}$ & $\begin{array}{l}\text { Nicky ficou triste (ou brabo, etc.). } \\
\text { Nicky ficou feliz (ou bem, agradecido etc.). }\end{array}$ & $\begin{array}{l}0 \\
1\end{array}$ \\
\hline Relação com benfeitor & $\begin{array}{l}\text { Não } \\
\text { Sim }\end{array}$ & $\begin{array}{l}\text { Porque ela achou a gatinha. } \\
\text { Porque tia Anne ajudou ela achar a gatinha. }\end{array}$ & $\begin{array}{l}0 \\
1\end{array}$ \\
\hline Deve ajudar & $\begin{array}{l}\text { Não } \\
\text { Sim }\end{array}$ & $\begin{array}{l}\text { Não. } \\
\text { Sim. }\end{array}$ & $\begin{array}{l}0 \\
1\end{array}$ \\
\hline Tipos de justificativa & $\begin{array}{l}\text { Não dever } \\
\text { Hábito } \\
\text { Consequência } \\
\text { Retribuir o favor }\end{array}$ & $\begin{array}{l}\text { Porque ela jogou o bolo fora. } \\
\text { Porque é isto o que as pessoas fazem. } \\
\text { Se não, ela vai demorar muito tempo. } \\
\text { Porque tia Anne ajudou ela achar a gatinha. }\end{array}$ & $\begin{array}{l}0 \\
1 \\
2 \\
3\end{array}$ \\
\hline \multicolumn{4}{|c|}{ História do Sweater } \\
\hline Sentimentospositivos & $\begin{array}{l}\text { Não } \\
\text { Sim }\end{array}$ & $\begin{array}{l}\text { Danielle ficou triste (ou com medo, etc). } \\
\text { Danielle ficou feliz (ou alegre, etc). }\end{array}$ & $\begin{array}{l}0 \\
1\end{array}$ \\
\hline Relação com benfeitor & $\begin{array}{l}\text { Não } \\
\text { Sim }\end{array}$ & $\begin{array}{l}\text { Porque ela tinha um sweater. } \\
\text { Porque Jane deu o sweater para ela. }\end{array}$ & $\begin{array}{l}0 \\
1\end{array}$ \\
\hline Deve emprestar & $\begin{array}{l}\text { Não } \\
\text { Sim }\end{array}$ & $\begin{array}{l}\text { Não. } \\
\text { Sim. }\end{array}$ & $\begin{array}{l}0 \\
1\end{array}$ \\
\hline Tipos de justificativa & $\begin{array}{l}\text { Não dever } \\
\text { Hábito } \\
\text { Consequência } \\
\text { Retribuir o favor }\end{array}$ & $\begin{array}{l}\text { Porque é dela. } \\
\text { Porque se usa a mesma tesoura. } \\
\text { Porque a professora vai xingar Jane. } \\
\text { Porque Jane deixou Danielle usar o sweater. }\end{array}$ & $\begin{array}{l}0 \\
1 \\
2 \\
3\end{array}$ \\
\hline
\end{tabular}

Alguns participantes não foram incluídos nas análises, porque eles disseram que o beneficiário deveria ajudar o benfeitor, mas não deram uma justificativa (responderam, por exemplo: "Eu não sei" ou "Eu não tenho uma razão para isso."). Na história da gatinha, 38 crianças responderam desta maneira e 12 , na história do sweater (alguns participantes deram esse tipo de justificativa em ambas as histórias e outros, em apenas uma delas).

Neste estudo, apresentaram-se as tarefas sempre na mesma ordem: (a) consideração da perspectiva visual, (b) crença falsa de segunda ordem, (c) crença falsa de primeira ordem, (d) história da gatinha e (e) história do sweater. 


\section{Resultados}

Examinou-se, inicialmente, se haveria diferenças significativas entre meninos e meninas quanto ao desenvolvimento de uma teoria da mente e compreensão da gratidão. Uma Análise Multivariada de Variância (MANOVA) revelou que, nas cinco medidas utilizadas (três de teoria da mente e duas de gratidão), não houve diferença significativa entre os sexos, Wilks' Lambda $F(5,176)=1,49$, $p<0,20$, confirmando assim a hipótese 1 . Desta forma, examinaram-se os dados conjuntamente nas análises subsequentes.

A seguir, testou-se a hipótese sobre a existência de uma relação entre a compreensão da gratidão e o desenvolvimento de uma teoria da mente, utilizando-se o coeficiente de Pearson. Como pode ser visto na Tabela 2, o padrão das correlações é claro: As crianças que tiveram um melhor desempenho nas tarefas sobre teoria da mente tiveram um desempenho significativamente melhor nas histórias sobre gratidão, confirmando a hipótese 2 .

Tabela 2

Síntese das Médias, Desvios Padrão e Correlações de Pearson dos Resultados das Tarefas sobre Gratidão e Teoria da Mente

\begin{tabular}{lccccccc}
\hline Tarefa & $M(D P)$ & 1 & 2 & 3 & 4 & 5 & $N$ \\
\hline 1. História da gatinha & $3,31(1,38)$ & - & $0,33^{* * *}$ & $0,26^{* * *}$ & $0,22^{* *}$ & $0,19^{*}$ & 190 \\
2. História do sweater & $3,67(1,82)$ & & - & $0,27^{* * *}$ & $0,28^{* * *}$ & $0,26^{* * *}$ & 216 \\
3. Crença falsa de primeira ordem & $3,25(1,11)$ & & & - & $0,20^{* *}$ & $0,49^{* * *}$ & 226 \\
4. Perspectiva visual & $1,94(1,12)$ & & & - & $0,14^{*}$ & 227 \\
5. Crença falsa de segunda ordem & $1,43(0,69)$ & & & & - & 227 \\
\hline
\end{tabular}

$* p<0,05, * * p<0,01, * * * p<0,001$.

Assim, examinou-se também a terceira hipótese: Uma teoria da mente é condição necessária para a compreensão da gratidão. Para isto, escolheu-se a tarefa de crença falsa de segunda ordem, a qual avalia o desenvolvimento de uma teoria da mente em um nível mais sofisticado, porque, em nossa amostra, apenas nove crianças não demonstraram qualquer evidência de uma teoria da mente na tarefa de crença falsa de primeira ordem. Este número era insuficiente para testar adequadamente essa hipótese.

Testou-se a terceira hipótese duas vezes. Em primeiro lugar, para verificar se uma completa evidência de uma teoria da mente é condição necessária para a compreensão da gratidão e, em segundo lugar, se uma evidência parcial de uma teoria da mente é condição necessária para tal. Neste estudo, quando a criança respondeu corretamente às duas questões relevantes da tarefa de crença falsa de segunda ordem, considerou-se que ela tinha uma completa evidência de uma teoria da mente; quando ela respondeu corretamente apenas a uma das questões, considerou-se que ela tinha uma evidência parcial. Definiu-se como evidência de que a criança tinha compreensão da gratidão, quando sua justificativa para ajudar o benfeitor incluiu a ideia de retribuir o favor. Utilizou-se o teste Quiquadrado para analisar se as crianças que não tinham uma completa evidência de uma teoria da mente nessa tarefa (não respondendo corretamente às duas questões relevantes) eram as que também não tinham uma compreensão da gratidão.

Os resultados indicaram que aquelas crianças que não tinham uma completa evidência de uma teoria da mente na tarefa de crença falsa de segunda ordem eram também as que mais tinham mostrado não ter uma compreensão da gratidão, na história da gatinha, $\chi^{2}(1)=11,22, p<$ 0,001 , e na história do sweater, $\chi^{2}(1)=6,44, p<0,02$. Todavia, na Tabela 3 , pode-se observar que $5,3 \%$ das crianças, na história da gatinha, e 4,6\% das crianças, na história do sweater, que não tinham uma completa evidência de uma teoria da mente mostraram que tinham uma compreensão da gratidão. Isto significa que uma completa evidência de uma teoria da mente na tarefa de crença falsa de segunda ordem não é condição necessária para a compreensão da gratidão.

Assim sendo, buscou-se verificar se pelo menos uma evidência parcial de uma teoria da mente na tarefa de crença falsa de segunda ordem (respondendo corretamente pelo menos uma das duas questões relevantes) não seria uma condição necessária para a compreensão da gratidão.

$\mathrm{Na}$ história da gatinha, os resultados indicaram que aquelas crianças que não tinham qualquer evidência de uma teoria da mente eram também as que mais tinham mostrado não ter uma compreensão da gratidão, $\chi^{2}(1)=$ $6,36, p<0,02$. Na Tabela 3, pode-se observar que as crianças que não tinham qualquer evidência de uma teoria da mente, na tarefa de crença falsa de segunda ordem, também não haviam compreendido a gratidão. Na história do sweater, porém, a relação entre evidência de uma teoria da mente na tarefa de crença falsa de segunda ordem e compreensão da gratidão não foi significativa, $\chi^{2}$ $(1)=3,60, p<0,06$. Nesta história, quase todas as crianças que não mostraram qualquer evidência de uma teoria 
Tabela 3

Número e Percentagem de Crianças com ou sem Evidência de uma Teoria da Mente (TOM) e Compreensão da Gratidão

\begin{tabular}{|c|c|c|}
\hline Compreensão da Gratidão & TOM Completa & Sem ou Parcial TOM \\
\hline & História da Gatinha & \\
\hline Gratidão & $34(17,0 \%)$ & $10(5,3 \%)$ \\
\hline \multirow[t]{2}{*}{ Sem gratidão } & $71(37,4 \%)$ & $75(39,5 \%)$ \\
\hline & História do Sweater & \\
\hline Gratidão & $28(13,0 \%)$ & $10(4,6 \%)$ \\
\hline \multirow[t]{2}{*}{ Sem gratidão } & $91(42,1 \%)$ & $87(40,3 \%)$ \\
\hline & História da Gatinha & \\
\hline Gratidão & $44(23,16 \%)$ & $0(0 \%)$ \\
\hline \multirow[t]{2}{*}{ Sem gratidão } & $127(66,84 \%)$ & $19(10 \%)$ \\
\hline & História do Sweater & \\
\hline Gratidão & $37(17,13 \%)$ & $1(0,46 \%)$ \\
\hline Sem gratidão & $154(71,3 \%)$ & $24(11,11 \%)$ \\
\hline
\end{tabular}

da mente também não tinham compreensão da gratidão. No entanto, pode-se observar na Tabela 3 que uma criança que não mostrou qualquer evidência de uma teoria da mente na tarefa de crença falsa de segunda ordem mostrou ter compreendido a gratidão. Isto significa que, neste estudo, a hipótese de que uma teoria da mente é condição necessária para a compreensão da gratidão não foi confirmada.

\section{Discussão}

Neste artigo, examinaram-se as relações entre a compreensão da gratidão e uma teoria da mente em crianças pré-escolares. Existem ainda poucos estudos sobre a gratidão na infância, embora resultados de pesquisa indiquem que as crianças são capazes de vivenciar (Gordon et al., 2004), expressar (Baumgarten-Tramer, 1938; Freitas et al., 2011) e compreender a gratidão (Freitas et al., 2009a, 2009b; Graham, 1988; Graham \& Barker, 1990; Harris et al., 1987; Castro et al., 2011; Russell \& Paris, 1994). Alguns autores (e.g., de Rosnay et al., 2004; Harwood \& Farrar, 2006; Heerey et al., 2003; Racine et al., 2007) investigaram as relações entre uma teoria da mente e a compreensão de emoções e sentimentos, mas nenhum havia abordado especificamente a gratidão.

É plausível pensar-se que existe uma relação entre gratidão e teoria da mente, uma vez que a avaliação da intenção do benfeitor parece ser um componente-chave da gratidão (Bonnie \& de Waal, 2004; Freitas et al., 2009a; Graham, 1988; McConnell, 1993). McAdams e Bauer (2004) levantaram a hipótese (mas não a testaram) de que uma teoria da mente é uma condição necessária para a gratidão.

Assim, neste estudo, inicialmente, buscou-se verificar se haveria diferença entre meninos e meninas, tanto no que diz respeito à compreensão da gratidão quanto ao desenvolvimento de uma teoria da mente. A hipótese de que não se encontraria essa diferença foi confirmada. É possível que haja uma diferença entre meninos e meninas quanto ao conteúdo da gratidão - a que eles e elas são gratos -, uma vez que, conforme sugeriram Gordon et al. (2004), as crianças são gratas àquilo que elas valorizam. Segundo esses mesmos autores, o fato de que, em seu estudo, as meninas se referiram mais a pessoas, enquanto os meninos mencionaram mais objetos, poderia ser explicado em função da maior importância atribuída às relações interpessoais pelas meninas. Outros estudos sobre aquilo a que as crianças são gratas são necessários para se verificar a pertinência desta explicação. É importante assinalar que em pesquisas sobre a capacidade das crianças e jovens de vivenciar (Froh et al., 2009) e de expressar (Freitas et al., 2011) a gratidão também não se encontraram diferenças significativas entre meninos e meninas. Em outras palavras, não se encontraram diferenças de gênero em todos os aspectos da gratidão investigados até este momento, mas apenas em relação ao seu conteúdo, no estudo de Gordon et al. (2004).

A seguir, testou-se a hipótese de que haveria uma relação entre a compreensão da gratidão e o desenvolvimento de uma teoria da mente. Esta hipótese foi confirmada. Aquelas crianças que tiveram um melhor desempenho nas tarefas sobre teoria da mente também mostraram ter uma melhor compreensão da gratidão. Isto faz sentido, visto que a gratidão requer que, de alguma forma, se leve em conta o ponto de vista do outro. $\mathrm{O}$ beneficiário sente-se feliz, quando recebe um benefício, mas é essencial que ele compreenda o papel do benfeitor, valorize-o e, como resultado, sinta uma dívida simbólica e uma necessidade de retribuir-lhe o favor. Para isto, uma teoria da mente é uma habilidade importante.

O desenvolvimento de uma teoria da mente seria condição necessária para a gratidão, como propuseram McAdams e Bauer (2004)? Considerando-se esta ideia, 
examinou-se a hipótese de que uma teoria da mente seria condição necessária para a compreensão da gratidão. Em primeiro lugar, buscou-se verificar se uma completa evidência de uma teoria da mente (a criança deveria responder corretamente as duas questões relevantes da tarefa de crença falsa de segunda ordem) seria condição necessária para a compreensão da gratidão. Esta hipótese não foi confirmada, visto que algumas crianças que não tinham uma completa evidência de uma teoria da mente haviam compreendido a gratidão. Em segundo lugar, utilizou-se uma evidência parcial de uma teoria da mente, segundo a qual a criança precisava ter respondido pelo menos uma das questões relevantes na tarefa de crença falsa de segunda ordem. Os resultados deste estudo indicaram que, embora a maioria das crianças que não mostrou qualquer evidência de uma teoria da mente na tarefa de crença falsa de segunda ordem também não compreendeu a gratidão, uma criança mesmo sem ter mostrado sequer uma evidência parcial de uma teoria da mente nessa tarefa mostrou ter compreendido a gratidão. Dito de outra maneira, neste estudo, a hipótese de que o desenvolvimento de uma teoria da mente seria condição necessária para a compreensão da gratidão não encontrou suficiente suporte empírico.

É importante ressaltar que o desenvolvimento de uma teoria da mente não é uma condição suficiente para a compreensão da gratidão. Muitas crianças que mostraram alguma evidência (completa ou parcial) de uma teoria da mente não compreenderam a gratidão. No entanto, um certo número dessas crianças de 5 anos mostrou ter uma compreensão da gratidão, o que vai ao encontro da ideia defendida por alguns autores (Graham \& Barker, 1990; Russell \& Paris, 1994) de que mesmo crianças pré-escolares são capazes de entender a gratidão.

Mais pesquisas são ainda necessárias para que se investigue o desenvolvimento da gratidão em crianças, abordando sob que condições ela emerge e como se desenvolve ao longo da infância. Futuras pesquisas deveriam incluir crianças com menos idade e, assim, poder-se-ia utilizar a tarefa de crença falsa de primeira ordem para testar a hipótese de que o desenvolvimento de uma teoria da mente seria condição necessária para a compreensão da gratidão. Estudos longitudinais seriam também importantes para se entender o desenvolvimento de outros processos psicológicos implicados na compreensão da gratidão, uma vez que os resultados deste estudo indicaram que uma teoria da mente não é suficiente para essa compreensão. Além disto, a realização de estudos transculturais seria fundamental para a investigação de possíveis efeitos culturais na compreensão da gratidão. Uma das lacunas na literatura recente sobre gratidão, segundo Watkins, van Gelder e Frias (2009), é justamente a consideração de suas prováveis variações culturais. Assim, os resultados deste estudo devem ser tomados com cautela, visto que são representativos de um grupo cultural específico.

\section{Referências}

Bartsch, K., \& Wellman, H. M. (1995). Children talk about the mind. New York: Oxford University Press.

Baumgarten-Tramer, F. (1938). "Gratefulness" in children and young people. Journal of Genetic Psychology, 53, 53-66.

Becker, J. A., \& Smenner, P. C. (1986). The spontaneous use of thank you by preschoolers as a function of sex, socioeconomic status, and listener status. Language in Society, 15(4), 537-545.

Blankson, A. N., O’Brien, M., Leerkes, E. M., Marcovitch, S., \& Calkins, S. D. (in press). Developmental dynamics of emotion and cognition processes in preschoolers. Social Development.

Bonnie, K. E., \& de Wall, F. B. M. (2004). Primate social reciprocity and the origin of gratitude. In R. A. Emmons \& M. E. McCullough (Eds.), The Psychology of Gratitude (pp. 213-229). New York: Oxford University Press.

Bono, G., \& Froh, J. J. (2009). Gratitude in school: Benefits to students and schools. In R. Gilman, E. S. Huebner, \& M. Furlong (Eds.), Handbook of Positive Psychology in Schools (pp. 77-88). New York: Routledge.

Castro, F., Rava, P. G. S., Hoefelmann, T. B., Pieta, M. A. M., \& Freitas, L. B. L. (2011). Deve-se retribuir? Gratidão e dívida simbólica na infância. Estudos de Psicologia (Natal). 16(1), 75-82.

Cutting, A. L., \& Dunn, J. (1999). Theory of mind, emotion understanding, language, and family background: Individual differences and interrelations. Child Development, 70, 853-865

de Rosnay, M., Pons, F., Harris, P. L., \& Morrell, J. M. B. (2004). A lag between understanding false belief and emotion attribution in young children: Relationships with linguistic ability and mothers' mental-state language. British Journal of Developmental Psychology, 22, 197-218.

Flavell, J. H., Everett, B. A., Croft, K., \& Flavell, E. R. (1981). Young children's knowledge about visual perception: Further evidence for the Level 1-Level 2 distinction. Developmental Psychology, 17, 99-103.

Flavell, J. H., Miller, P. H., \& Miller, S. A. (2002). Cognitive development $\left(4^{\text {th }}\right.$ ed.). Upper Saddle River, NJ: Prentice-Hall.

Freitas, L. B. L., Pieta, M. A. M., \& Tudge, J. R. H. (2011). Beyond politeness: The expression of gratitude in children and adolescents. Psicologia: Reflexão e Crítica, 24(4), $757-$ 764

Freitas, L. B. L., Silveira, P. G., \& Pieta, M. A. M. (2009a) Sentimento de gratidão em crianças de 5 a 12 anos. Psicologia em Estudo, 14(2), 243-250.

Freitas, L. B. L., Silveira, P. G., \& Pieta, M. A. M. (2009b). Um estudo sobre o desenvolvimento de gratidão na infância. Revista Interamericana de Psicologia, 43(1), 49-56.

Froh, J. J. (2010, April). Teaching children how to think gratefully: Unveiling a new gratitude intervention for youth Paper presented at the Conference on Human Development, New York City.

Froh, J. J., \& Bono, G. (2008). The gratitude of youth. In S. J. Lopez (Ed.), Positive Psychology: Exploring the best in people (Vol. 2, pp. 55-78). Westport, CT: Greenwood.

Froh, J. J., Kashdan, T. B., Ozimkowski, K. M., \& Miller, N. (2009). Who benefits the most from a gratitude intervention in children and adolescents? Examining positive affect as a moderator. The Journal of Positive Psychology, 4, 408422. 
Gleason, J. B., \& Weintraub, S. (1976). The acquisition of routines in child language. Language in Society, 5(2), 129136.

Gordon, A. K., Musher-Eizenman, D. R., Holub, S. C., \& Dalrymple, J. (2004). What are children thankful for? An archival analysis of gratitude before and after the attacks of September 11. Journal of Applied Developmental Psychology, 25(5), 541-553.

Graham, S. (1988). Children's developing understanding of the motivational role of affect: An attributional analysis. Cognitive Development, 3(1), 71-88.

Graham, S., \& Barker, G. P. (1990). The down side of help: An attributional-developmental analysis of helping behavior as a low-ability cue. Journal of Educational Psychology, 82(1), 7-14.

Harris, P. L., Olthof, T., Meerum Terwogt, M., \& Hardman, C. E. (1987). Children's knowledge of the situations that provoke emotion. International Journal of Behavioral Development, 10(3), 319-343.

Harwood, M. D., \& Farrar, M. J. (2006). Conflicting emotions: The connection between affective perspective taking and theory of mind. British Journal of Developmental Psychology, 24, 401-418.

Heerey, E. A., Keltner, D., \& Capps, L. M. (2003). Making sense of self-conscious emotion: Linking theory of mind and emotion in children with autism. Emotion, 3(4), 394-400.

Jou, G. I., \& Sperb, T. M. (1999). Teoria da mente: Diferentes abordagens. Psicologia: Reflexão e Crítica, 12(2), 287-306.

Komter, A. E. (2004). Gratitude and gift exchange. In R. A. Emmons, \& M. E. McCullough (Eds.), The Psychology of Gratitude (pp. 195-212). New York: Oxford University Press.

McAdams, D. P., \& Bauer, J. J. (2004). Gratitude in modern life: Its manifestations and development. In R. A. Emmons \& M. E. McCullough (Eds.), The Psychology of Gratitude (pp. 81-99). New York: Oxford University Press.

McConnell, T. (1993). Gratitude. Philadelphia, PA: Temple University Press.

McCullough, M. E., Kilpatrick, S. D., Emmons, R. A., \& Larson, D. B. (2001). Is gratitude a moral affect? Psychological Bulletin, 127(2), 249-266.

Perner, J., \& Wimmer, H. (1985) “John thinks that Mary thinks that...": Attribution of second-order beliefs by 5-to 10-yearold-children. Journal of Experimental Child Psychology, 39, 437-471.

Piaget, J. (1954). Les relations entre l'affectivité et l'intelligence. Paris: Sorbonne.

Piaget, J. (1977). Études sociologiques (3. ed.). Genève, Suisse: Droz. (Original work published 1965)

Piaget, J. (1992). Le jugement moral chez l'enfant (7. ed.). Paris: Presses Universitaires de France. (Original work published 1932)

Pieta, M. A. M., \& Freitas, L. B. L. (2009). Sobre a gratidão. Arquivos Brasileiros de Psicologia, 61(1), 100-108.

Racine, T. P., Carpendale, J. I. M., \& Turnbull, W. (2007). Parent-child talk and children's understanding of beliefs and emotions. Cognition and Emotion, 21(3), 480-494.

Roazzi, A., \& Santana, S. M. (2008). Teoria da mente e estados mentais de primeira e segunda ordem. Psicologia: Reflexão e Crítica, 21(3), 437-445.

Russell, J. A., \& Paris, F. A. (1994). Do children acquire concepts for complex emotions abruptly? International Journal of Behavioral Development, 17(2), 349-365.
Santana, S. M., \& Roazzi, A. (2006). Cognição social em crianças: Descobrindo a influência de crenças falsas e emoções no comportamento humano. Psicologia: Reflexão e Crítica, 19(1), 1-8.

Sperb, T. M., \& Maluf, M. R. (2009). Desenvolvimento sociocognitivo: Estudos brasileiros sobre "teoria da mente". São Paulo, SP: Vetor.

Sullivan, K., Zaitchik, D., \& Tager-Flusberg, H. (1994). Preschoolers can attribute second-order beliefs. Developmental Psychology, 30, 395-402.

Taylor, M. (1988). Conceptual perspective taking: Children's ability to distinguish what they know from what they see. Child Development, 59, 703-718.

Watkins, P. C., van Gelder, M., \& Frias, A. (2009). Furthering the science of gratitude. In S. J. Lopes, \& C. R. Snyder (Eds.), Oxford Handbook of Positive Psychology ( $2^{\text {nd }}$ ed., pp. 437445). London: Oxford University Press.

Wellman, H. M., \& Liu, D. (2004). Scaling of theory-of-mind tasks. Child Development, 75, 523-541. 